\title{
Nanoembossment of Au Patterns on Microspheres
}

Gang Zhang, Dayang Wang, ${ }^{*}$ and Helmuth Möhwald

${ }^{\dagger}$ Max Planck Institute of Colloids and Interfaces, D-14424, Potsdam, Germany

\section{Supporting Information:}
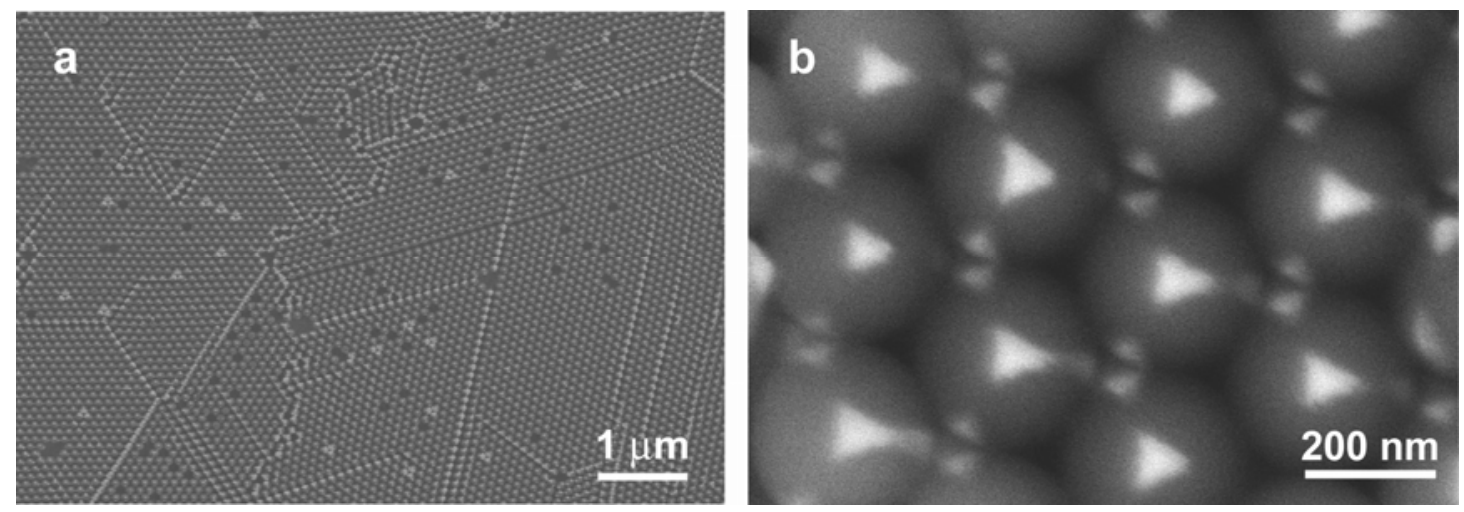

Figure S1. Low (a) and high (b) magnification SEM picture of $270 \mathrm{~nm}$ PS spheres with Au-patterned surfaces generated by templating the top monolayers of colloidal crystals with (111) facets parallel to the substrates.

\footnotetext{
* Author for correspondence. Fax: 49-331-5679202. Email: dayang.wang@mpikg-golm.mpg.de.
} 


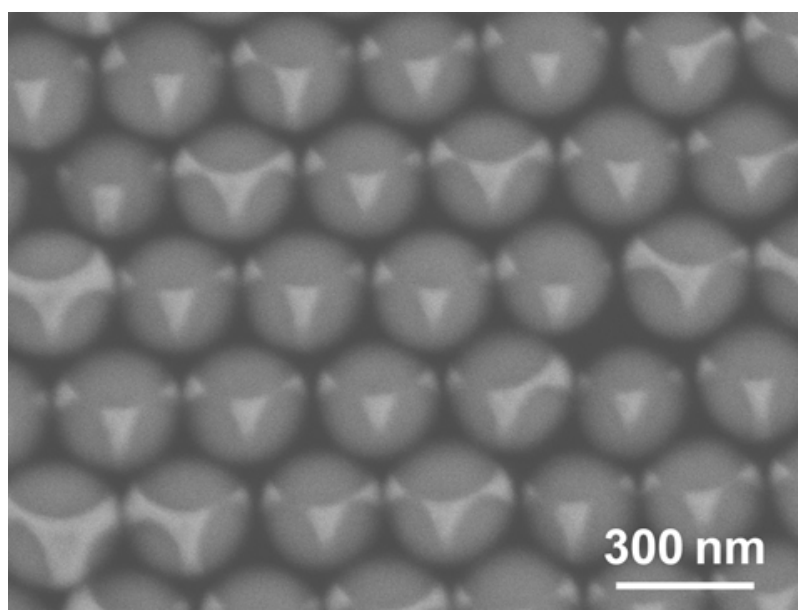

Figure S2. SEM picture of $296 \mathrm{~nm}$ silica spheres with Au-patterned surfaces generated by templating the top monolayers of colloidal crystals with the preferential crystal orientation of (111) parallel to substrates as masks. 


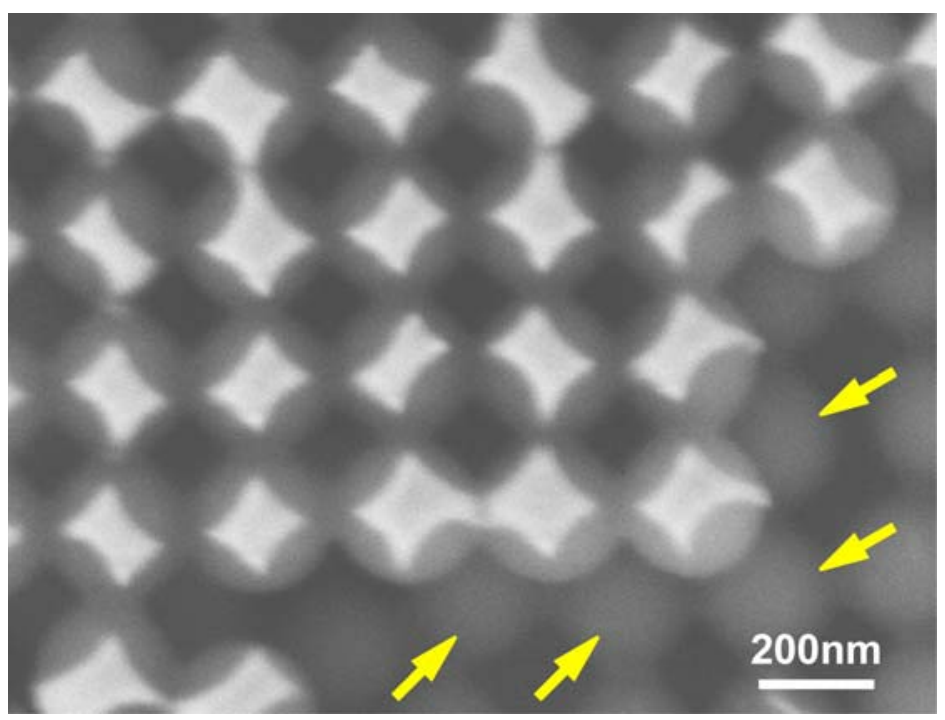

Figure S3. SEM picture of $270 \mathrm{~nm}$ PS spheres with Au-patterned surfaces generated by templating the upper double layers of the (100) facets of their colloidal crystals. In this case, the sphere of the third layer is located just beneath that of the first layer, no $\mathrm{Au}$ patterns were observed in the spheres in the third layer, indicated by yellow arrows. 


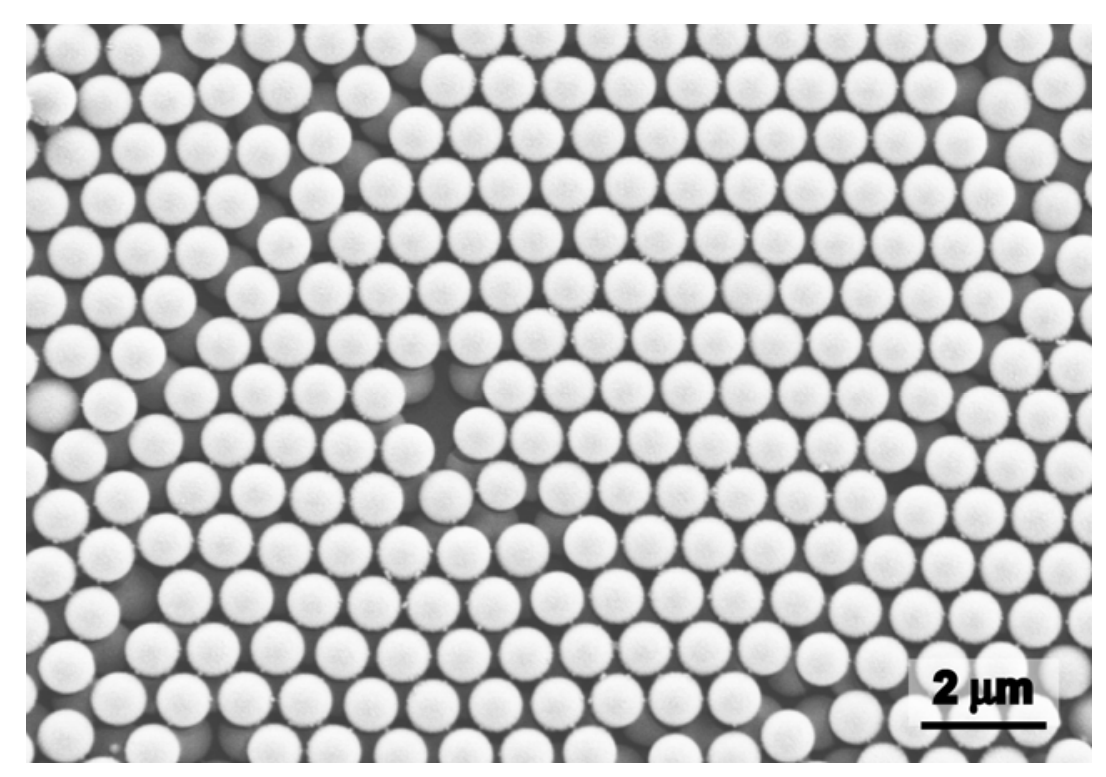

Figure S4. SEM image of gold - coated $925 \mathrm{~nm}$ PS colloidal crystals, etched by $\mathrm{O}_{2}-$ plasma for 5 min. 

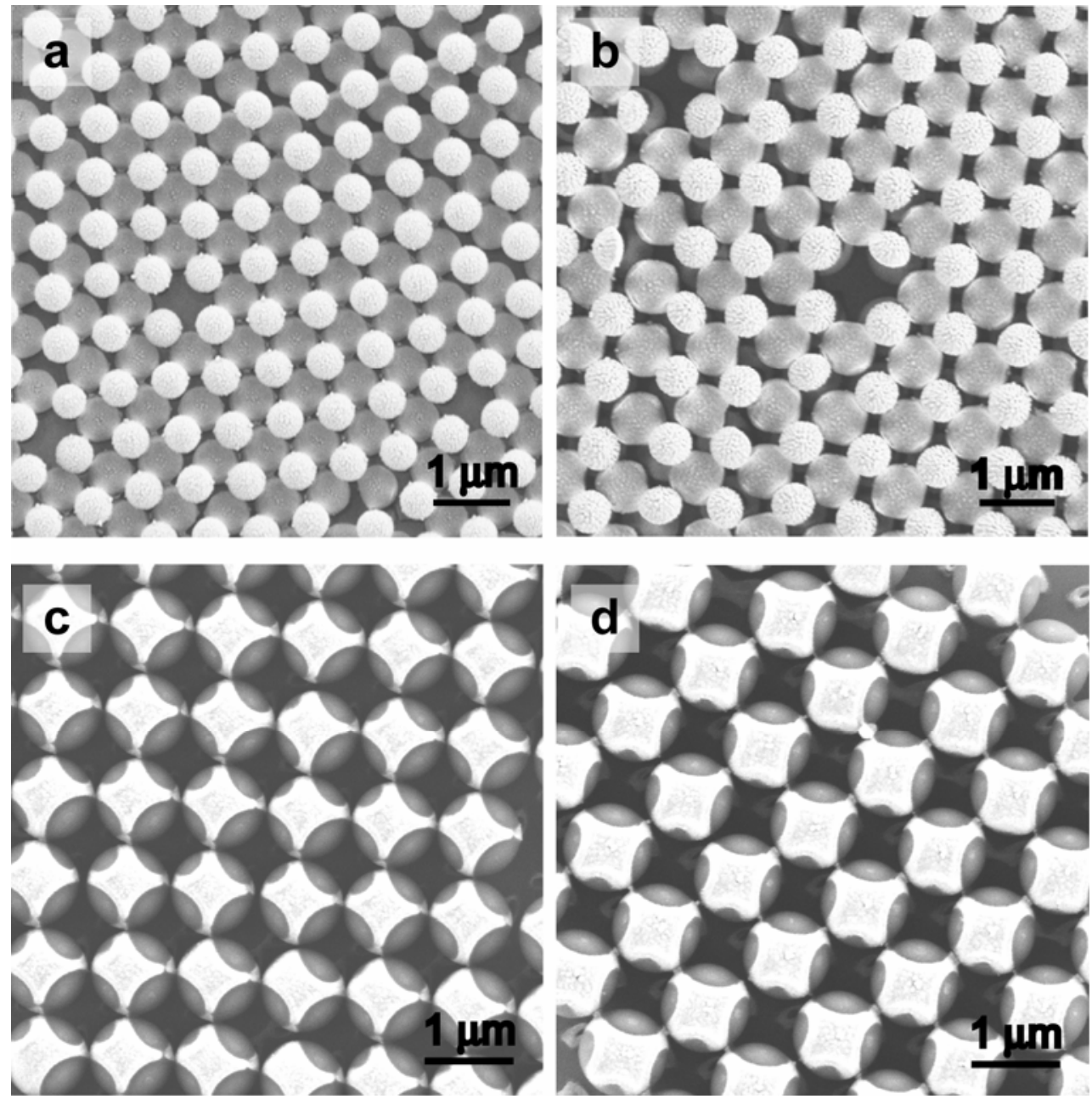

Figure S5. SEM images of gold - coated $925 \mathrm{~nm}$ PS colloidal crystals with (100) facets parallel to the substrates, etched by $\mathrm{O}_{2}$ - plasma for $10 \mathrm{~min}$ (a) and $20 \mathrm{~min}$ (b). The gold layers are $100 \mathrm{~nm}$ in thickness. After peeling off the top monolayers in these crystals, the corresponding SEM images of the Au patterns formed in the second spheres are shown in Figure c and d. 

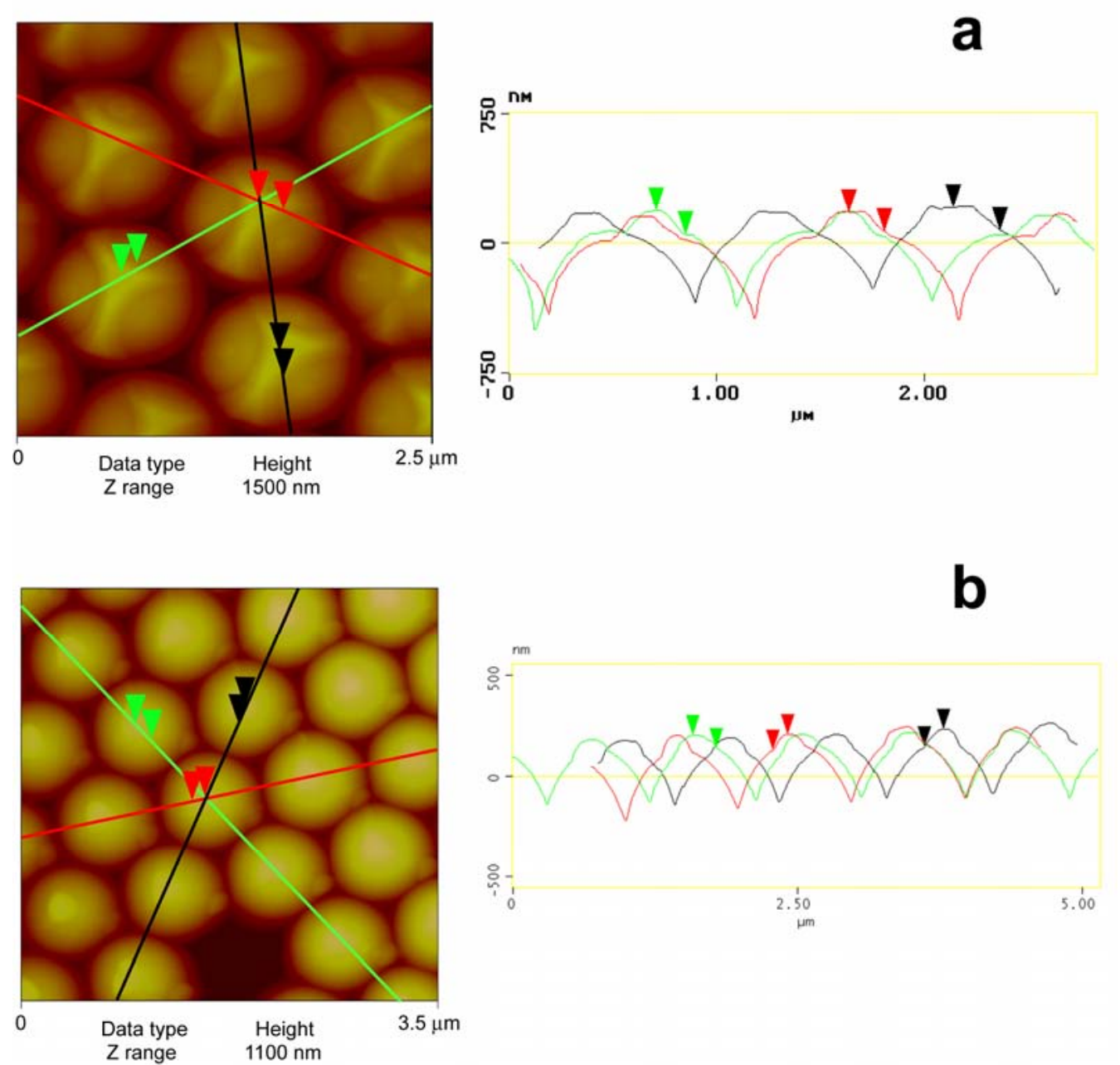

Figure S6. AFM height image of the Au nanodots on $925 \mathrm{~nm}$ PS spheres formed by templating the upper single (a) and double (b) layers of the colloidal crystals, etched by $\mathrm{O}_{2}$-plasma for $10 \mathrm{~min}$ and coated with a $100 \mathrm{~nm}$ Au layer. The stacking symmetry of the crystals is ABCABC and their (111) facets are parallel to the substrates. The corresponding SEM images are shown in Figure 5c and 6a. The cross-section profiles of these dots are shown in the right. Note that mainly due to the high curvature of the microsphere surface and the broadening effect of AFM tips, the Au nanodots shown here appear larger and not clear-cut as compared to those in their SEM images. 


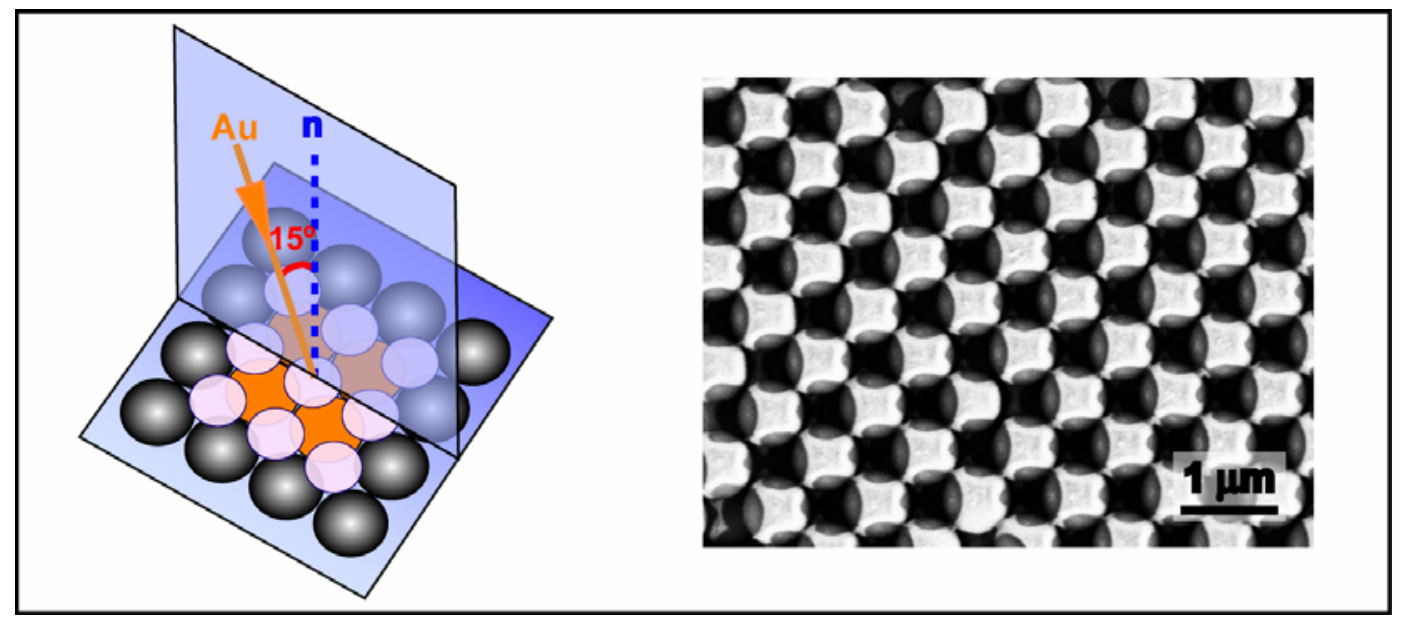

Figure S7. SEM image of 925 nm PS spheres with Au-patterned surfaces, obtained by using the top monolayers of (100) facets of 10 min plasma etched colloidal crystals as masks during Au vapor deposition at the incident angles, $\theta$ of $15^{\circ}$. 

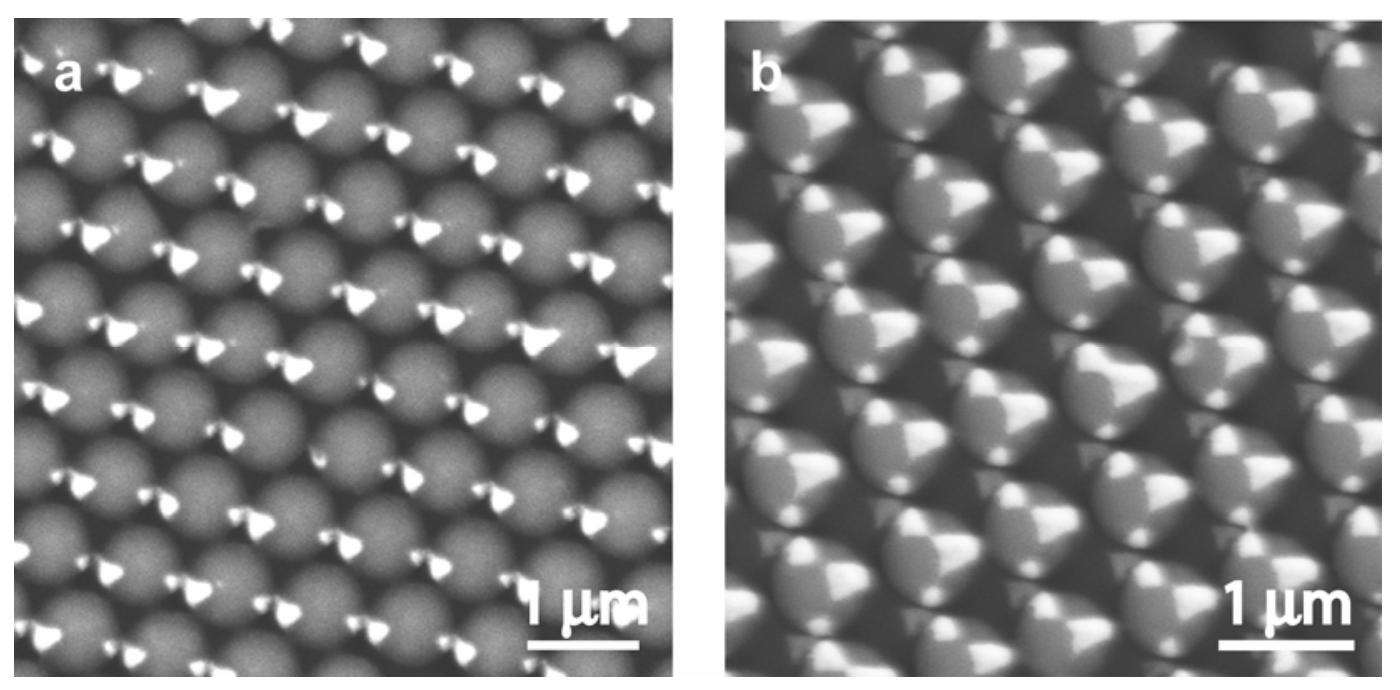

Figure S8. SEM images of $270 \mathrm{~nm}$ PS spheres with Au - patterned surfaces, obtained by using (111) facets of their colloidal crystals, etched by $\mathrm{O}_{2}$ - plasma for 3 min, with an ABAB (a) stacking symmetry, and by using (100) facets of their colloidal crystals as masks during Au vapor deposition at the incident angle of $10^{\circ}$. The gold layers are $100 \mathrm{~nm}$ in thickness. 
Modeling of Nanoembossment of Au Patterns on Microspheres.

a
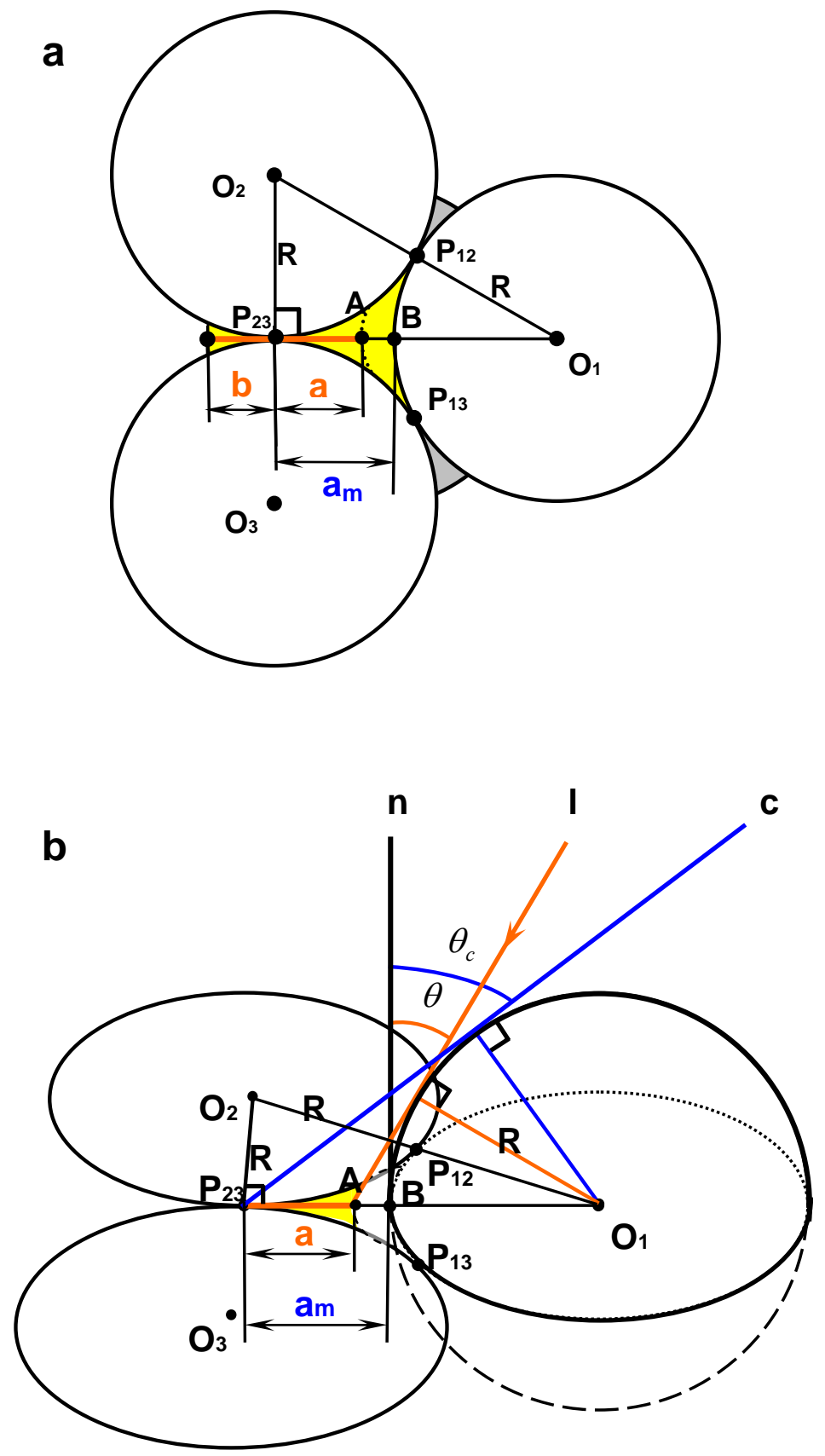

Figure S9 shows schematic depictions of the geometric feature of close-packed microspheres in a colloidal crystal. (a) is a top-view of the close-packed three spheres and (b) their quasi-3D illustration. $\boldsymbol{R}$ is their radius. $\boldsymbol{O}_{1}, \boldsymbol{O}_{2}$, and $\boldsymbol{O}_{3}$ are the centers of the three spheres, $\boldsymbol{O}_{\mathbf{4}}$ is the center of the sphere underneath, and $\boldsymbol{P}_{\mathbf{1 2}}, \boldsymbol{P}_{\mathbf{1 3}}, \boldsymbol{P}_{\mathbf{2 3}}$ are the points of intersection of three spheres. $\boldsymbol{n}$ points the normal direction of the equator 
plane of the three spheres. Herein we assume that the incident Au flow is restricted within the $\boldsymbol{O}_{\mathbf{1}} \boldsymbol{P}_{23}-\boldsymbol{n}$ plane. The orange line represents the incidence and the blue line the maximal incidence angle, $\theta_{c}$.

Assuming that there is no diffraction of the Au beam, the projection area of the $\mathrm{Au}$ flow, highlighted by yellow, should determine the dimension and shape of the pattern formed on the spheres underneath. The length between $\boldsymbol{B}$ and $\boldsymbol{P}_{23}$ points can represent the maximal size of the Au pattern obtained, $\boldsymbol{a}_{\boldsymbol{m}}$, at $\theta=0$. When $\theta>0$, the projection of the upper half of the top layer sphere on the interstices of the top layer has to be taken into account, decreasing the projection area of the Au flow and in turn leading to a smaller Au pattern. Accordingly, we used the length between $\boldsymbol{A}$ and $\boldsymbol{P}_{23}$ points, $\boldsymbol{a}$, to represent the sizes of the large Au domains obtained on the second layer sphere. $\boldsymbol{b}$ represent the size of the small Au domains at the edges of the second layer spheres. According to the geometry shown in Figure S9, one can derive " $\boldsymbol{a}$ " as follows:

$$
\begin{aligned}
& a=A P_{23}=O_{1} P_{23}-O_{1} A \\
& O_{1} P_{23}=\sqrt{(2 R)^{2}-R^{2}}=\sqrt{3} R \\
& O_{1} A=\frac{R}{\cos \theta} \\
& a=\sqrt{3} R-\frac{R}{\cos \theta}=R\left(\sqrt{3}-\frac{1}{\cos \theta}\right)
\end{aligned}
$$

When $\theta$ is 0 , the maximal value of $\boldsymbol{a}$ is achieved, $a_{m}=R(\sqrt{3}-1)$. Provided no Au pattern is formed on the second layer spheres, $\boldsymbol{a}$ equals zero. Thus, the critical value of q can be calculated by:

$$
\begin{aligned}
& \cos \theta_{c}=\frac{R}{O_{1} P_{23}}=\frac{1}{\sqrt{3}} \\
& \theta_{c}=\arccos \frac{1}{\sqrt{3}} \approx 54.74^{\circ}
\end{aligned}
$$


Furthermore, the value of $\mathrm{b}$, the size of the small Au domains located at the edge of the second layer spheres, can be derived by:

$$
\begin{aligned}
& b=P_{23} E=O_{4} E-O_{4} P_{23} \\
& O_{4} E=R \\
& O_{4} P_{23}=R \cdot \tan 30^{\circ}=R / \sqrt{3} \\
& b=R-R / \sqrt{3}=R\left(1-\frac{1}{\sqrt{3}}\right)
\end{aligned}
$$




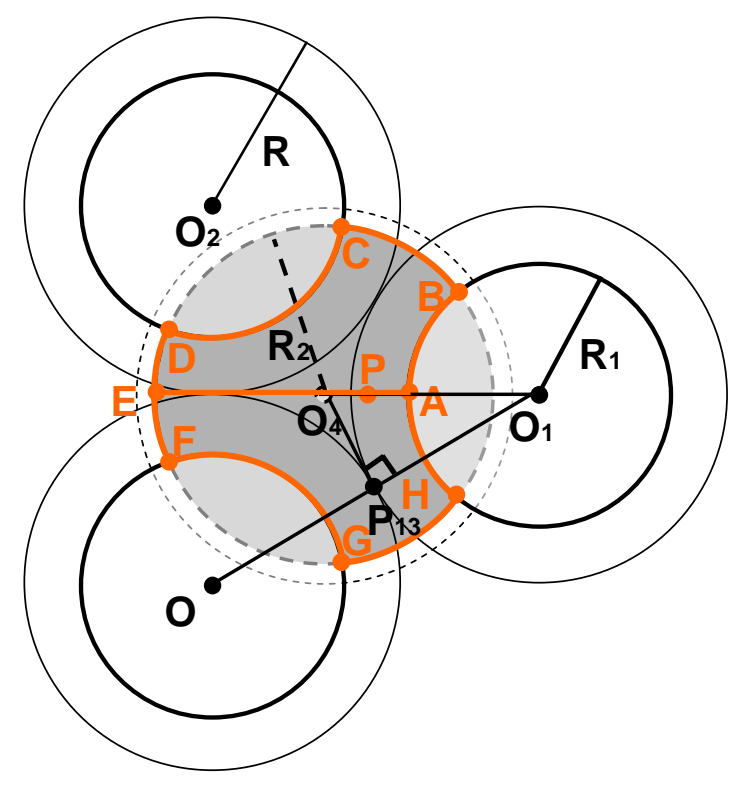

Figure S10 shows a top view of geometric feature of etched colloidal crystals. $\boldsymbol{R}_{\mathbf{1}}$ and $\boldsymbol{R}_{\mathbf{2}}$ are the radii of the top and second layer spheres, respectively, and $\boldsymbol{R}$ is the radius of original microspheres before $\mathrm{O}_{2}$ etching. The projection area of the Au flow is enclosed by the A-B-C-D-E-F-G-H orange curve, as suggested by the SEM pictures (Figures $5 \mathrm{c}$ and). The length between $\mathrm{A}$ and $\mathrm{E}$ points is used to represent the maxmal size $\left(\boldsymbol{a}_{p m}\right)$ of the Au pattern obtained at $\theta=0$. At $\theta>0$, the P-E length, $\boldsymbol{a}_{p}$, may represent the size of the pattern. The difference between the A-E and P-E lengths can be easily calculated following the way mentioned above.

Based on the geometry shown in Figure S10, we may gain the size of pattern on low sphere:

$$
\begin{aligned}
& a_{p}=P E=P O_{4}+O_{4} E \\
& O_{4} E=R_{2}
\end{aligned}
$$




$$
\mathrm{PO}_{4}=\mathrm{O}_{1} \mathrm{O}_{4}-\mathrm{O}_{1} P
$$

and,

$$
\begin{aligned}
& O_{1} O_{4}=\frac{R}{\sin 60^{\circ}}=\frac{2}{\sqrt{3}} R \\
& O_{1} P=\frac{R_{1}}{\cos \theta}
\end{aligned}
$$

by introducing (10), (11), (12), and (13) into (9), $\boldsymbol{a}_{p}$ can be calculated by:

$$
\begin{aligned}
& a_{p}=P E=R_{2}+\frac{2}{\sqrt{3}} R-\frac{R_{1}}{\cos \theta}=R\left(\frac{2}{\sqrt{3}}+\beta-\frac{\alpha}{\cos \theta}\right) \\
& \text { where } \alpha=\frac{R_{1}}{R} \text { and } \beta=\frac{R_{2}}{R} .
\end{aligned}
$$

When $\theta$ is $0, \boldsymbol{a}_{p}$ reaches the maximum, $a_{p m}=R\left(\frac{2}{\sqrt{3}}+\beta-\alpha\right)$.

When $\theta=\theta_{p c}, \boldsymbol{a}_{\boldsymbol{p}}$ equals zero, no Au patterns is formed. Thus,

$$
\begin{aligned}
& \frac{R_{1}}{\cos \theta_{p c}}=O_{1} E=O_{1} O_{4}+O_{4} E=\frac{2}{\sqrt{3}} R+R_{2} \\
& \theta_{p c}=\arccos \frac{R_{1}}{\frac{2}{\sqrt{3}} R+R_{2}}=\arccos \frac{3 \sqrt{3} \alpha}{2+3 \sqrt{3} \beta}
\end{aligned}
$$

When both $\alpha$ and $\beta$ equal to 1, Figure S10 should be identical to Figure S9. According to Equation E3, thus:

$$
a_{p}=R\left(\frac{2}{\sqrt{3}}+1-\frac{1}{\cos \theta}\right)
$$

This equation can be easily separated into: 


$$
\begin{aligned}
& a_{p}=R\left(\frac{2}{\sqrt{3}}+1-\frac{1}{\cos \theta}\right) \\
& =R\left(\frac{3-1}{\sqrt{3}}+1-\frac{1}{\cos \theta}\right)=R\left(\sqrt{3}-\frac{1}{\sqrt{3}}+1-\frac{1}{\cos \theta}\right) \\
& =R\left(\sqrt{3}-\frac{1}{\cos \theta}\right)+R\left(1-\frac{1}{\sqrt{3}}\right) \\
& =a+b
\end{aligned}
$$

Thus, Equation E3 derived from Figure S10 become identical to the sum of Equations E1 and E2 from Figure S9, which suggests a good consistency of our modeling albeit it is rather rough and simple. 